\title{
Epithelioid sarcoma presenting as the reflex sympathetic dystrophy syndrome
}

\author{
C.L. Summers and M. Shahi ${ }^{1}$
}

University Departments of Orthopaedics and ${ }^{\prime}$ Medicine, Manchester Royal Infirmary, Oxford Road, Manchester M139WL, UK.

\begin{abstract}
Summary: A case of reflex sympathetic dystrophy caused by an epithelioid sarcoma is presented. This is the first report of a local peripheral tumour associated with the reflex sympathetic dystrophy syndrome.
\end{abstract}

\section{Introduction}

Reflex sympathetic dystrophy is usually associated with minor trauma, fracture or surgical procedures on bones, or follows peripheral nerve injury. ${ }^{1}$ In the present report we describe an adult patient who developed sympathetic dystrophy in association with epithelioid sarcoma.

\section{Case report}

A 58 year old company director presented in 1983 with pain in the dorsum of his left foot. There was no history of injury. The pain increased and the foot became swollen. An initial diagnosis of gout was made but there was no response to anti-inflammatory drugs. A radiograph taken at that time showed no abnormality. Local exploratory surgery, through the dorsum of the foot, revealed fibrous tissue around the second and third metatarsal heads. Histology demonstrated a focal chronic inflammatory infiltrate.

In the 6 month post-operative period he developed more persistent pain in the metatarsal region with hyperhydrosis, and increasing oedema of the left foot consistent with a reflex sympathetic dystrophy syndrome. In the ensuing 18 months his symptoms persisted with only partial relief from various treatment regimes including high dose steroids, regional sympathetic blockade with guanethidine, chemical lumbar sympathectomy and calcitonin. He was referred to Manchester Royal Infirmary in 1985. Examination showed his left leg to be oedematous to below the knee with longitudinal ridged nails. There was a pigmented surgical scar with excessive sweating of the foot (Figure 1). Review of the serial radiographs showed progressive generalized osteopenia of the

Correspondence: M. Shahi, M.R.C.P., St Mary's Hospital, Praed Street, London W2 1NY, UK.

Accepted: 4 September 1986 bones of the left foot with progressive erosion of the distal regions of the shafts of the second and third metatarsals (Figures 2 and 3). Isotope bone scan showed increased uptake in the left tibia and foot. A computed tomographic (CT) scan (1.5 mm sections) of the left foot confirmed the osteopenia and demonstrated an abnormal soft tissue mass (approximately

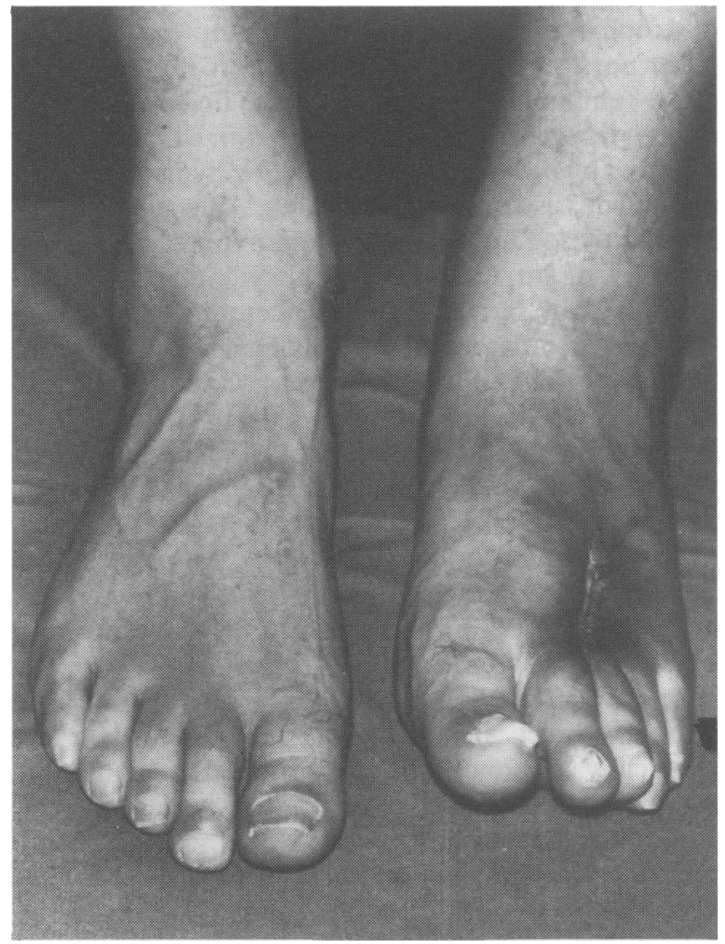

Figure 1 Reflex sympathetic dystrophy of the left foot showing oedema, hyperhydrosis and ridging of the nails.

(C) The Fellowship of Postgraduate Medicine, 1987 
$2 \mathrm{~cm}$ in diameter) lying below and adjacent to the eroded second metatarsal head (Figure 4). Nerve conduction studies showed no evidence of a peripheral nerve lesion.

A surgical lumbar sympathectomy was performed which abolished the hyperpathia and hyperhydrosis, but he continued to experience severe pain in the dorsum of the left foot. Because of the severity of his pain and the finding of a mass on CT scan, a further exploratory operation was performed from the plantar aspect of the foot. This confirmed erosion of the second and third metatarsal heads with adjacent yellow friable material, resembling caseation. Histology revealed the features of an epithelioid sarcoma (Figure 5). A lymph node resected from the left groin showed reactive hyperplasia only and no evidence of metastasis. A below-knee amputation was performed three weeks later; histology showed the tumour to be localized and invading an adjacent digital nerve. Six months later he remains well and free of recurrence.

\section{Discussion}

This is the first reported case of a local peripheral tumour associated with reflex sympathetic dystrophy. Although central tumours have been previously reported $^{2,3}$ their association with the reflex sympathetic dystrophy syndrome may have been either coincidental or possibly due to a non-metastatic manifestation of the tumour. Characteristically the first symptom of reflex sympathetic dystrophy is pain which is soon followed by oedema and then later by hyperaemia and hyperhydrosis of the affected area. ${ }^{4}$ The syndrome is commonly self limiting, but often requires therapy in the form of surgical or chemical sympathectomy. ${ }^{5}$

Epithelioid sarcoma is an uncommon soft tissue tumour, first described by Enzinger in $1970 .{ }^{6}$ The tumour usually occurs in young adults, and the extremities of the upper limbs are the principal sites of involvement. Presentation is that of a slow-growing, painless, firm nodule which often becomes ulcerated; pain or tenderness is seldom a prominent feature and has only been reported when the tumour encroaches on a nerve. ${ }^{6}$ Radiological features have been described by Lo et al. in $1977 ; ;^{7}$ There may be a soft tissue mass with associated erosion or thickening of adjacent bone. Occasionally destruction of bone occurs through direct invasion by the tumour. ${ }^{8.9}$ Histologically the tumour may be mistaken for an inflammatory process or granuloma, but on careful inspection, the tumour cells have a distinct nodular arrangement and a tendency to undergo central degeneration and necrosis; the cells are epithelioid in appearance and eosinophilic. The tumour has a propensity for local and late distal metastasis and adequate treatment requires radical amputation of the

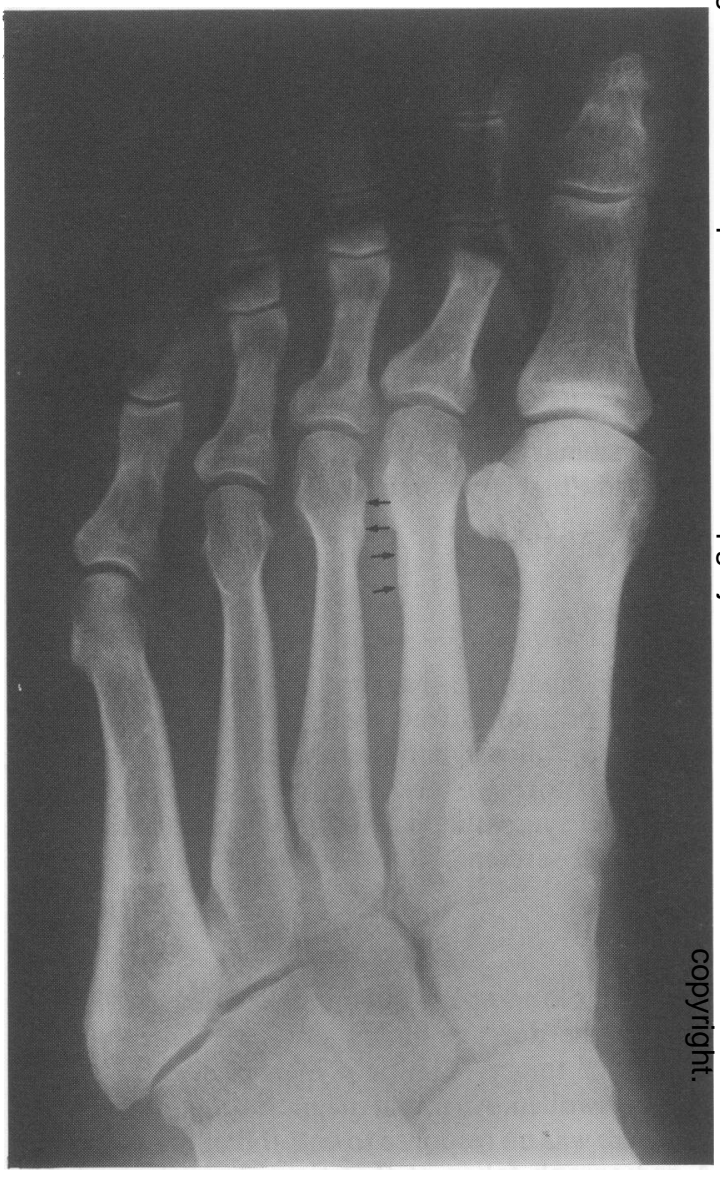

Figure 2 Oblique radiograph of the left foot (May 1983) 6 months after initial presentation shows periosteal reaction along the distal shafts of the second and third metatarsals (arrows).

affected part of the limb.

The correct diagnosis was masked in our patient because the reflex sympathetic dystrophy had been $\stackrel{0}{0}$ attributed to the initial exploratory operation. The persistence of pain after the sympathectomy and the $\mathrm{O}$ presence of an abnormal soft tissue mass on CT scan $D$ were important factors in the decision to undertake further exploratory surgery, which led to the correct $N$ diagnosis. Pain is an uncommon feature of epithelioid $\mathrm{Gr}$ sarcoma and, in retrospect, the presenting symptoms $N$ of pain followed by oedema, could have been the first $\underset{\omega}{N}$ manifestations of reflex sympathetic dystrophy associated with the tumour. The postulated mechan- 0 ism is that the tumour compressed or invaded a mixed $\Phi$ digital nerve trunk and produced an artificial (or $\stackrel{\mathscr{C}}{+}$ ephaptic) synapse between efferent sympathetic and ${ }^{\circ}$ afferent sensory fibres. Continual crossover of efferent $\stackrel{\vec{D}}{\stackrel{\mathrm{D}}{\mathrm{d}}}$ 


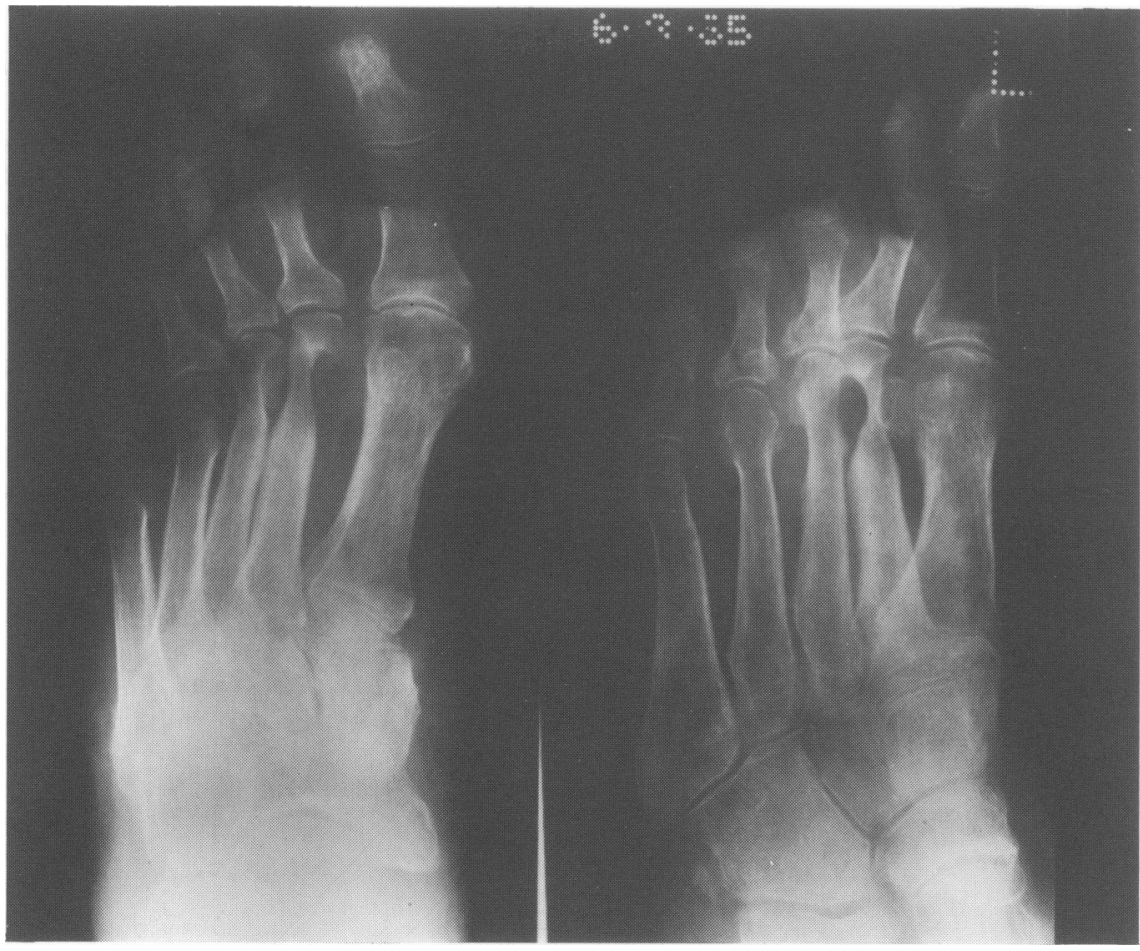

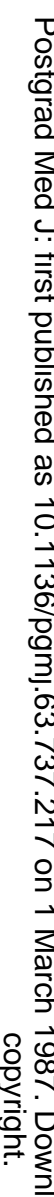

Figure 3 Radiographs of the left foot (August 1985). In addition to the generalized osteopenia which has developed as a result of the reflex sympathetic dystrophy, there is now severe pressure erosion of the distal ends of the second and third metatarsals in the region where there was previously periosteal reaction.

sympathetic impulses down afferent sensory fibres further facilitated the painful afferent somatic impulses and the release of vasomotor substances resulting in the syndrome of reflex sympathetic dystrophy. ${ }^{10}$

We conclude that in patients with reflex sympathetic dystrophy, when no obvious underlying cause is discernible, an underlying neoplasm should be considered.

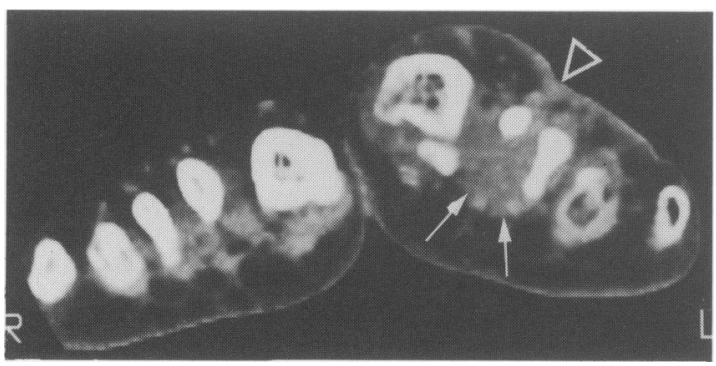

Figure 4 CT scan (1.5 mm section) shows the soft tissue mass (arrows) lying below and adjacent to the eroded distal shafts of the second and third metatarsals of the left foot. The surgical scar on the dorsum of the foot is also evident (open triangle).

\section{Acknowledgements}

We would like to thank Professor Adams, Dr Adams, Dr Schady and Mr Maltby for their valuable assistance in the writing of this manuscript.

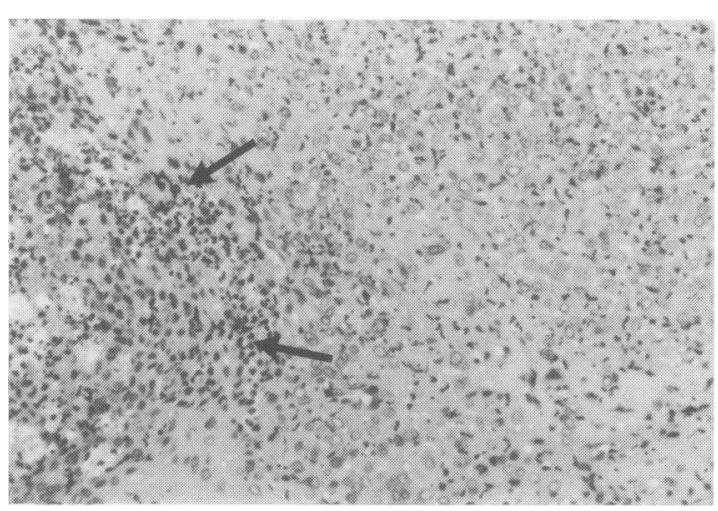

Figure 5 Epithelioid sarcoma. Part of the tumour nodule showing central necrosis (arrows) mimicking a necrotizing granulomatous process (haematoxylin eosin $\times 100)$. 


\section{References}

1. Imanuel, H.M., Levy, F.L. \& Geldwert, J.J. Sudeck's atrophy: A review of the literature. J Foot Surg 1981, 4: 243-246.

2. Kozin, F., McCarty, D.J., Sims, J. et al. The reflex sympathetic dystrophy syndrome. Am J Med 1976, 60: $321-331$.

3. Subbarao, J. \& Stillwell, G.K. Reflex sympathetic dystrophy of the upper extremity: analysis of total outcome of management of 125 cases. Arch Phys Med Rehabil 1981, 62: 549-554.

4. Pak, T.J., Martin, G.M., Magness, J.L. et al. Reflex sympathetic dystrophy: review of 140 cases. Minn Med 1970, 53: 507-512.

5. Bonica, J.J. Causalgia and other reflex sympathetic dystrophies. Postgrad Med 1973, 53: 143-148.

6. Enzinger, F.H. Epithelioid sarcoma; a sarcoma simulat-

ing a granuloma or carcinoma. Cancer 1970, 26: 1029 1035.

7. Lo, H.H.., Kalisher, L. \& Faix, J.D. Epithelioid sar $\overrightarrow{\bar{c}}$ coma: radiologic and pathologic manifestations. Am Roentgenol 1977, 128: 1017-1020.

8. Bryan, R.S., Soule, E.H., Dobyns, J.E. et al. Primary epithelioid sarcoma of the hand and forearm. J. Bone Joint Surg 1974, 56A: 458-461.

9. Prat, J., Woodruff, J.M. \& Marcove, R.C.: Epithelioidu sarcoma; an analysis of 22 cases indicating the prognostic significance of vascular invasion and regional lymph $\vec{\circ}$ node metastasis. Cancer 1978, 41: 1472-1487.

10. Drucker, W.R., Hubay, C.A., Holden, W.D. et al. Path ogenesis of post traumatic sympathetic dystrophy. Am Surg 1959, 97: 454-461. 